\title{
High Precision Photometry from EulerCam and TRAPPIST: The Case of WASP-42, WASP-49 and WASP-50
}

\author{
Monika Lendl ${ }^{1}$, Michaël Gillon ${ }^{2}$ and Didier Queloz ${ }^{1}$ \\ ${ }^{1}$ Université de Genève, \\ Chemin des Maillettes 51, 1290 Sauverny, Switzerland \\ email: monika.lendl@unige.ch \\ ${ }^{2}$ Université de Liège, Allée du 6 août 17, Sart Tilman, Liège 1, Belgium
}

\begin{abstract}
Transiting extrasolar planets provide unmatched insights into the structure and composition of close-in planets. When a planet transits its host star, its radius is known, which together with radial velocity measurements, allows accessing the planetary density. We present results obtained using the Euler and TRAPPIST telescopes that aim at reaching very high accuracy on the parameters derived from transit lightcurves. Here, we show the case of the recently discovered WASP-42b and WASP-49b and new observations of WASP-50b.
\end{abstract}

Keywords. planetary systems - stars: individual: WASP-42 - stars: individual: WASP-49 stars: individual: WASP-50 - techniques: photometric

\section{Approach}

Ground-based photometric observations of extrasolar planet transits and occultations are known to suffer from correlated noise on timescales similar to the timescales of interest (Pont et al. 2006). Precisely these effects have been a limiting factor on the groundbased detection and evaluation of atmospheric features through transit and occultation lightcurves.

Our approach to mitigate these effects consists in observing not one expensive highprecision lightcurve but obtaining several "cheaper" ground-based lightcurves and combining them. For this purpose we make use of EulerCam (Lendl et al. 2012) at the $1.2 \mathrm{~m}$ Euler-Swiss telescope and the automated 0.6m TRAPPIST telescope (Jehin et al. 2011) that are both installed at the La Silla site. The precision reached is similar to the one obtained for unique transits observed with $8 \mathrm{~m}$ class telescopes.

\section{Observations and Modeling}

The presented EulerCam observations were done using an $r^{\prime}$-Gunn filter while TRAPPIST used a combined $I+z^{\prime}$ filter. We apply a small defocus to the telescopes in order to improve the duty cycle and spread the light over more pixels. Details on the EulerCam observation and reduction procedures are described in Lendl et al. (2012).

All lightcurves are modeled using the MCMC code described in Gillon et al. (2012). For each transit, we find a combined solution for the transit and the photometric baseline for which we test models including parameters such as pixel shifts, sky background and seeing variations. To obtain the final results, all transits are modeled together with radialvelocity data obtained with CORALIE [e.g. Ségransan et al. (2010)]. 


\section{WASP-42b and WASP-49b}

WASP-42b is a $M_{P}=0.500 \pm 0.035 M_{J}, R_{P}=1.080 \pm 0.057 R_{J}$ planet on a 5 -day orbit around a K1 star (Lendl et al. 2012). While the planet is no outlier in terms of mass and radius, it is remarkable as it has a slightly eccentric orbit, having a small but significant eccentricity of $e=0.060 \pm 0.013$.

WASP-49b is slightly more massive than Saturn $\left(M_{P}=0.378 \pm 0.027 M_{J}, R_{P}=\right.$ $1.115 \pm 0.047 R_{J}$ ), orbiting a G6 star every 2.7 days (Lendl et al. 2012). With a metallicity of $[\mathrm{Fe} / \mathrm{H}]=0.36 \pm 0.05$, WASP-49 is one of the most metal poor planet hosts known. The planet has a radius larger than predicted from models [e.g. Fortney et al. (2007)], and thus forms part of the ensemble of inflated hot giant planets.

Both planets have been characterized from EulerCam and TRAPPIST lightcurves, that are displayed in Figure 1.
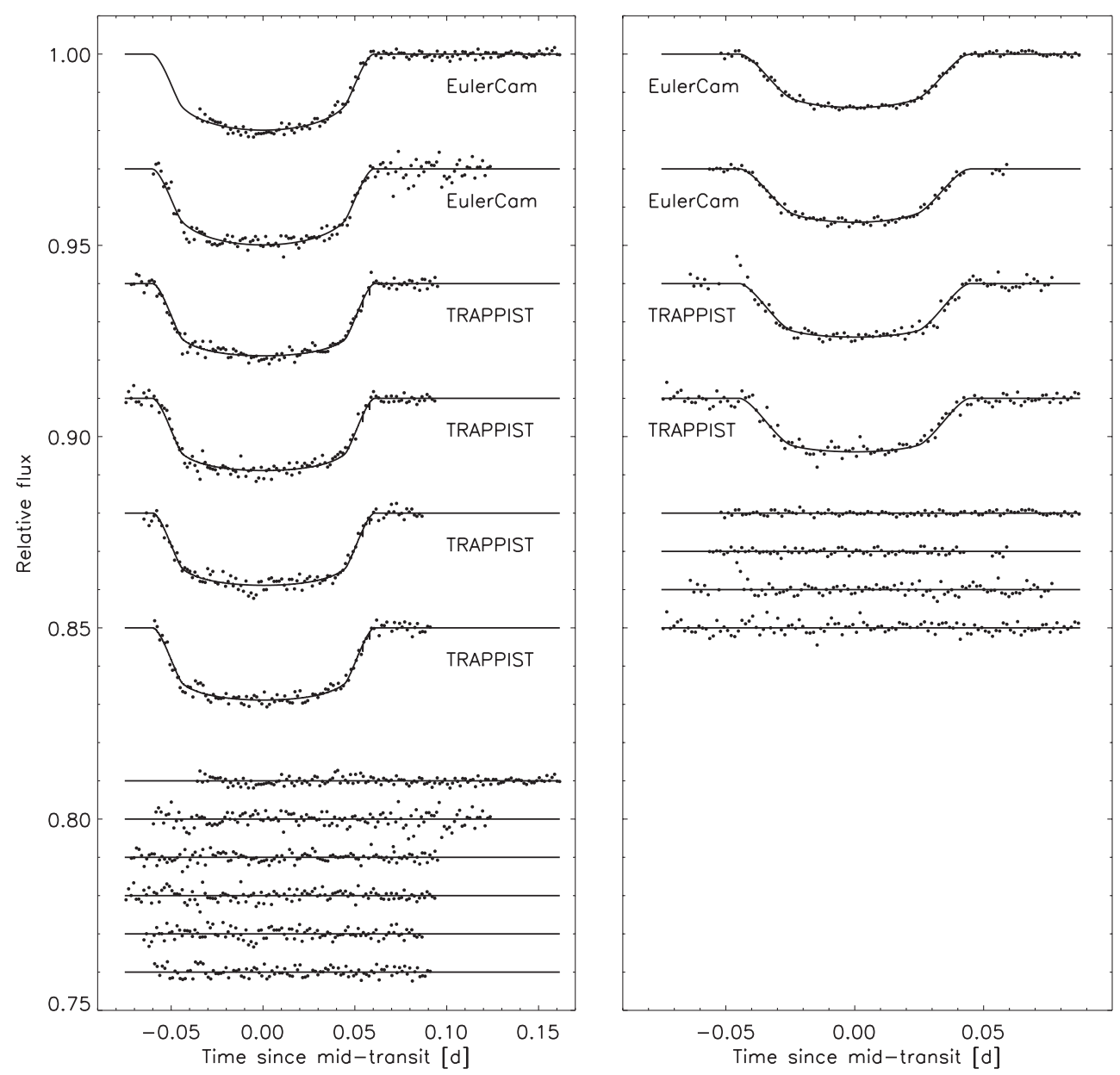

Figure 1. All lightcurves of WASP-42 (left) and WASP-49 (right) obtained with EulerCam and TRAPPIST. The data have been binned into two minute intervals for display purposes, and the residuals are shown in the lower part of the plots. 


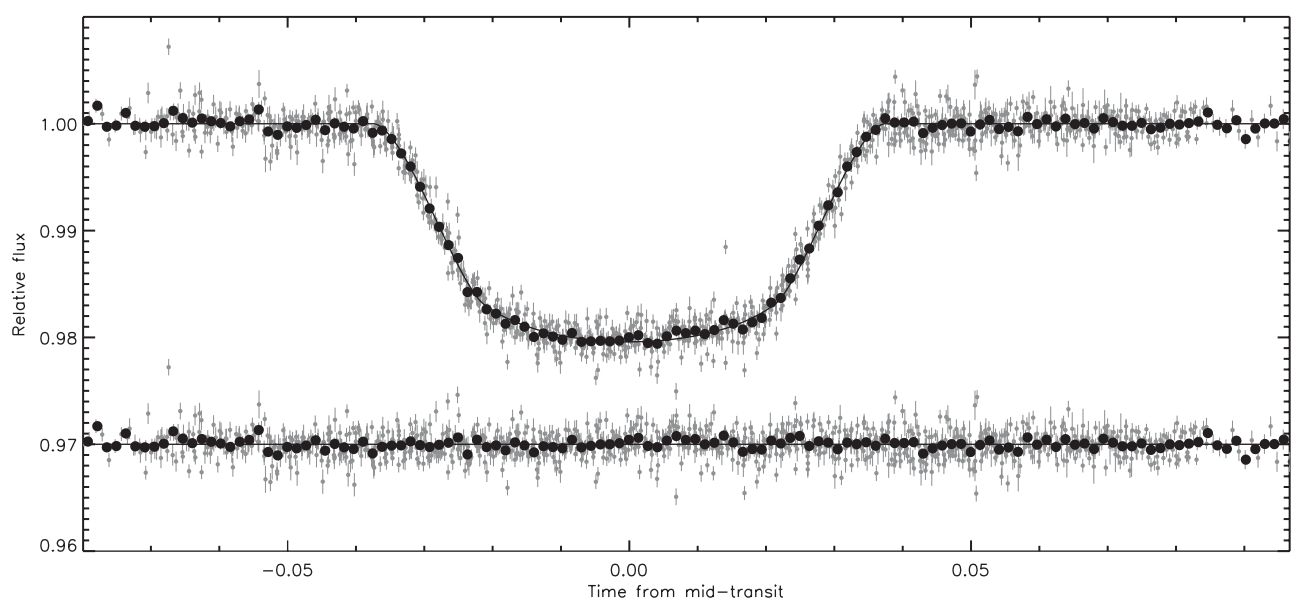

Figure 2. The combined lightcurve of WASP-50b consisting of seven single EulerCam observation sequences. The phased individual measurements are shown in grey, while the black points show the data binned into five minute intervals.

\section{WASP-50b}

WASP-50b (Gillon et al. 2011) is a $M_{P}=1.47 \pm 0.09 M_{J}, R_{P}=1.15 \pm 0.05 R_{J}$ planet in a 2-day orbit around an active G6 star. In contrast to many known hot Jupiters, WASP-50b does not show an inflated radius, having a density near that of Jupiter.

We have observed seven transits of WASP-50 with EulerCam, as part of a campaign to study this system in detail. While each lightcurve has an RMS in the order of 1 mmag (per 2 minute bin), we obtain a very clean transit lightcurve by combining all our data. The combined lightcurve is shown in Figure 2, yielding an RMS of $496 \mathrm{ppm}$ if a bin size of 2 minutes is used.

\section{Conclusion}

We increase precision and accuracy of the measured transit shape through combining many high-precision lightcurves from dedicated instruments. This way, using moderate ground based facilities, we aim at characterizing transiting planets in detail.

\section{References}

Gillon, M., Doyle, A. P., Lendl et al. 2011, A\&3A, 533, A88

Gillon, M., Triaud, A. H. M. J., Fortney, J. J. et al. 2012, A\&A, 541, A4

Fortney, J., Marley, M. S., \& Barnes, J. W., 2007 ApJ, 659, 1661

Jehin, E., Gillon, M., Queloz, D. et al. 2011 The Messenger, 145, 2

Lendl, M., Anderson, D. R., Collier-Cameron, A. et al. 2012 A 6 A, 544, A72

Pont, F., Zucker, S., \& Queloz, D. 2006 MNRAS, 373, 231

Sato, B., Fischer, D., Henry, G. et al. 2005 ApJ, 633, 465

Ségransan, D., Udry, S., Mayor, M. et al. 2010 A $\& A$, 511, 45 